\title{
Multi-scale coupling simulation in directional solidification of superalloy based on cellular automaton-finite difference method
}

\author{
Zhao Guo, * Jian-xin Zhou, Ya-jun Yin, Dong-qiao Zhang, Xiao-yuan Ji and Xu Shen \\ State Key Laboratory of Materials Processing and Die \& Mould Technology, Huazhong University of Science and Technology, Wuhan 430074, \\ China
}

\begin{abstract}
Casting microstructure evolution is difficult to describe quantitatively by only a separate simulation of dendrite scale or grain scale, and the numerical simulation of these two scales is difficult to render compatible. A three-dimensional cellular automaton model couplling both dendritic scale and grain scale is developed to simulate the microstructure evolution of the nickel-based single crystal superalloy DD406. Besides, a macro-mesoscopic/ microscopic coupling solution algorithm is proposed to improve computational efficiency. The simulation results of dendrite growth and grain growth of the alloy are obtained and compared with the results given in previous reports. The results show that the primary dendritic arm spacing and secondary dendritic arm spacing of the dendritic growth are consistent with the theoretical and experimental results. The mesoscopic grain simulation can be used to obtain results similar to those of microscopic dendrites simulation. It is indicated that the developed model is feasible and effective.
\end{abstract}

Key words: multi-scale coupling; dendritic growth; grain growth; directional solidification; cellular automata; numerical simulation

CLC numbers: TP391.99 Document code: A

Article ID: 1672-6421(2017)05-398-07

$\mathrm{D}$ rectional solidification technology is one of the key technologies for manufacturing high performance and complex parts such as aero engines and gas turbines blades ${ }^{[1-6]}$. In the manufacturing process of blades, microstructure defects such as misoriented grains and freckles are easily formed, which seriously reduce the overall performance of the blades. The solidification simulation method can help to analyze the mechanism of the microstructure evolution and optimize the solidification process parameters during casting.

At present, the most widely used simulation methods for solidification microstructure include the Volumeaveraged, the Phase Field, the Monte Carlo and the Cellular Automaton (CA) methods ${ }^{[7]}$. Among them, the CA method has the advantages of clearly describing the physical meaning and high computational efficiency of solidification phase transition, and has been widely used

\footnotetext{
*Jian-xin Zhou

Male, born in 1975, Professor, Ph.D. His research interests mainly focus on computer applications in foundry industry, especially casting process simulation and smart manufacturing for foundry enterprises.

E-mail: zhoujianxin@mail.hust.edu.cn
}

Received: 2017-08-15; Accepted: 2017-08-28 in solidification microstructure simulation at the mesograin and micro-dendritic scale.

In multi-scale solidification microstructure simulation, the macroscopic temperature and the microstructure evolution of solidification are generally calculated by using the coupled method of the CA method and the finite element (FE) method or the finite difference (FD) method. For instance, based on CA-FE method, the 3D decentered square method, proposed by Rappaz and Gandin et al. ${ }^{[8-11]}$, was used to simulate the directional solidification process of the blade. Then, based on the front tracking method, the CA-FE method was modified to solve the heat flow problem in the solidification mushy zone of casting. Similarly, based on the commercial casting simulation software ProCAST, the numerical simulation of the directional solidification process of the single crystal superalloy was conducted by Carter et al. ${ }^{[12]}$ and Meng et al. ${ }^{[13]}$. In addition, the CA-FD method was also developed by $\mathrm{Xu}$ et al. ${ }^{[14]}$ to simulate the directional solidification process of the superalloy turbine blades.

It is important to note that the CA method mentioned above is only applied to the mesoscopic grain scale. The CA model of mesoscopic grain scale is based on 
the theory of solidification interface stability. It is the solution of the dendritic growth velocity, the tip radius of the dendrites and the supercooling degree of the interface, ignoring the dendritic morphology of the grain itself; the numerical calculation is very fast. For the CA model of the microscopic dendritic scale, solving the solidification interface growth rate ${ }^{[15]}$ is based on the solute or energy conservation relationship at the front of the dendritic growth interface, taking full account of the influence of the solute composition and interface curvature on the dendritic morphology ${ }^{[16-19]}$, and the numerical solution is very accurate. However, the former is based on the analytical model, and the latter is based on conservation model. It is difficult to couple the two in solidification simulation.

Accordingly, a typical nickel-based single crystal superalloy DD406 was studied. Based on the advantages of the analysis model and the conservation model, a three-dimensional CA model coupling dendrite scale and grain size is developed. First of all, considering the solute undercooling, curvature undercooling and other factors, the analytical model of the dendrite tip growth velocity in front of the interface is used to calculate the solid fraction. Secondly, the modified decentered square method was used to calculate the growth rate of arbitrary dendritic/grain interface, and the solid fraction in the interface was coupled with the modified decentered square method to track the interface. Finally, the 3D CA model proposed in this study is compared with the theoretical and experimental results given in previous studies. Combined with the actual macro scale flow, the effect of different directional solidification parameters on the microstructure evolution of the superalloy DD406 was studied.

\section{Model and governing equations}

\subsection{Macroscopic model of heat transfer}

The governing equation for macroscopic temperature field is given by

$$
\rho c_{p} \frac{\partial T}{\partial t}=\frac{\partial}{\partial x}\left(k_{i} \frac{\partial T}{\partial x}\right)+\frac{\partial}{\partial y}\left(k_{i} \frac{\partial T}{\partial y}\right)+\frac{\partial}{\partial z}\left(k_{i} \frac{\partial T}{\partial z}\right)-\rho L \frac{\partial F_{S}}{\partial t}+Q_{R}
$$

where $\rho$ is density, $c_{p}$ is the specific heat capacity, $T$ is the temperature, $t$ is the solidification time, $k_{i}$ is the thermal conductivity, $L$ is the solidification latent heat, $F_{S}$ is the solid fraction, and $Q_{R}$ is the radiant heat source during the directional solidification process.

In addition, for the calculation of the radiation heat source, the method of High Rate Solidification (HRS) in the directional solidification is taken as an example to simplify the radiation heat transfer between the heating zone, the cooling zone and the simulated calculation area in the furnace. The heat transfer between any two finite gray bodies is calculated by StefanBoltzman's law. The governing equation is given by

$$
Q_{R}=\sigma\left(T_{1}^{4}-T_{2}^{4}\right) /\left(\frac{1-\varepsilon_{1}}{\varepsilon_{1} A_{1}}+\frac{1-\varepsilon_{2}}{\varepsilon_{2} A_{2}}+\frac{1}{A_{1} X_{1,2}}\right)
$$

where $\sigma$ is the Stefan-Boltzmann radiation constant, $T_{1}, T_{2}$ are the temperature of any two finite gray bodies, and $\varepsilon_{1}, \varepsilon_{2}$ are the emissivity, and $A_{1}, A_{2}$ are the occupied boundary surface area, their values equal to the area of the grid unit, and $X_{1,2}$ is the radiation angle factor, the value is taken as constant 1 .

\subsection{Meso-microscopic model of dendritic and grain evolution}

In the solidification microstructure simulation, the continuous nucleation model proposed by Rappaz and Gandin et al. ${ }^{[20]}$ is used to describe the random nucleation process of dendrites/ grains, as shown in Eq. (3):

$$
n(\Delta T)=\int_{0}^{\Delta T} \frac{n_{\max }}{\sqrt{2} \pi \Delta T_{\sigma}} \exp \left[-\frac{1}{2}\left(\frac{\Delta T-\Delta T_{N}}{\Delta T_{\sigma}}\right)^{2}\right] d\left(\Delta T^{\prime}\right)
$$

where $\Delta T$ is the undercooling, $n(\Delta T)$ is the nucleation number density at $\Delta T, n_{\max }$ is the initial nucleation base number, $\Delta T_{\sigma}$ is the undercooling variance in nucleation, $\Delta T_{N}$ is the undercooling mean in nucleation, respectively.

The growth rate of the dendritic interface and the non-dendritic interface are calculated by using a unified and simplified analytical model proposed by Nastac and Stefanescu ${ }^{[21]}$. At the same time, considering the influence of solute component supercooling and curvature on the growth rate of dendrites in the simulation, the phase equilibrium equation and the solute diffusion equation at the interface are integrated to calculate. The main governing equations of the model are given by

$$
\begin{aligned}
& V=\left(\frac{\Gamma \pi^{2} m\left(k_{0}-1\right) C_{L}^{*}}{D_{L}}\right)^{-1} \Delta T^{2} \\
& T_{L}^{e q}=T_{0}^{e q}+m\left(C_{L}^{*}-C_{0}\right)-\Gamma K
\end{aligned}
$$

$\frac{\partial C_{L}}{\partial t}=\frac{\partial}{\partial x}\left(D_{L} \frac{\partial C_{L}}{\partial x}\right)+\frac{\partial}{\partial y}\left(D_{L} \frac{\partial C_{L}}{\partial y}\right)+\frac{\partial}{\partial z}\left(D_{L} \frac{\partial C_{L}}{\partial z}\right)+C_{L}\left(1-k_{0}\right) \frac{\partial f_{S}}{\partial t}$

where $V$ is the interface growth rate, $\Gamma$ is the Gibbs-Thomson coefficient, $m$ is the liquidus slope, $k_{0}$ is the partition coefficient, $C_{0}$ is the initial composition at the temperature $T_{0}^{\mathrm{eq}}, C_{L}{ }^{*}$ is the interface equilibrium composition at the temperature $T_{L}^{\mathrm{eq}}, D_{L}$ is the solute diffusion coefficient in liquid, $f_{S}$ is the solid fraction, $K$ is the curvature of the interface calculated by an approximate method $^{[18]}$.

\section{Numerical algorithm and solution procedures}

\subsection{Macro-mesoscopic/microscopic coupling solution}

The CA-FD method is used to simulate the directional solidification process of superalloy. Two sets of uniform numerical grid $\Delta X, \Delta x(\Delta x=\Delta X / 10)$ are adopted, with the same time step $\Delta t$. For the microscopic scale simulation, the cell 
states are defined as three types: liquid $\left(f_{s}=0\right)$, solid $\left(f_{s}=1\right)$ or interface $\left(0<f_{s}<1\right)$. And the cell neighbours are characterized by the first-order neighbours (6 neighbourhoods), second-order neighbours (12 neighbourhoods) and third-order neighbours (8 neighbourhoods)

The solid phase fraction of mesoscopic/microscopic cell ${ }^{[22]}$ is calculated as Eq. (7):

$$
\Delta f_{S}=\frac{V \Delta t}{\sqrt{2} \Delta x}
$$

In order to ensure the convergence of the numerical calculation, the solid phase fraction of the cell is limited to 1 in a time step $\Delta t$, that is to say, the solidification interface is no more than one unit mesh.

The solid phase fraction of macroscopic cell is calculated as
Eq. (8):

$$
\Delta F_{S}=\frac{\sum \Delta f_{S}^{i}}{(\Delta X / \Delta x)^{3}}
$$

In the first step, the macroscopic temperature field is calculated by the finite difference method. Secondly, the macroscopic temperature field is mapped to the temperature field of the mesoscopic/microscopic cell by the linear interpolation method. Then, the dendritic growth rate and solid fraction are calculated by the supercooling functions. Finally, the solid fraction of the mesoscopic/microscopic cells are mapped back to the solid fraction of the macroscopic cell, accompanied by the release of latent heat, which affects the distribution of the macroscopic temperature field. The macro-mesoscopic/ microscopic coupling solution is shown in Fig. 1.

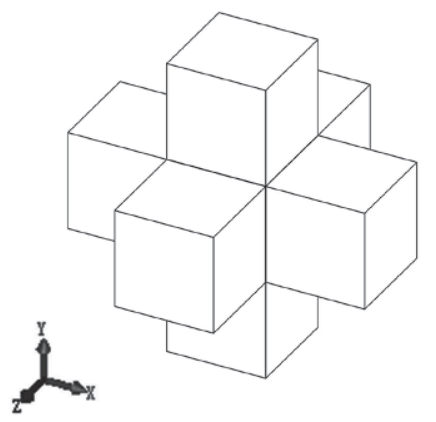

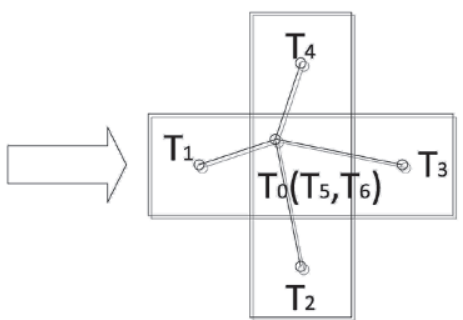

$T_{0}=T\left(T_{1}, T_{2}, T_{3}, T_{4}, T_{5}, T_{6}\right)$

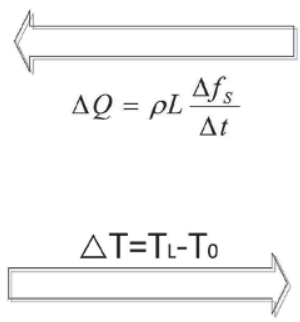

$\triangle f_{s}=f_{s}(t+\triangle t)-f_{s}(t)$

Fig. 1: Macro-mesoscopic / microscopic coupling solution

\subsection{Cell transformation or capture rules in cellular automaton}

The modified decentered square method is adopted. The general algorithm is used to track the six vertices of the octahedron. When one of six vertices touches the liquid cell in the surrounding neighbor, it is considered that the neighbor liquid cell is captured and transformed into the interface cell. At the same time, the growth center of the new interface cell is calculated from the decentered square growth of the initial interface cell growth center. In time step $\Delta t$, the six vertices of the octahedron may touch one of the surrounding 26 neighbors' liquid cells, and even touch two neighbor liquid cells, resulting in the complexity of decentered square algorithm. In order to avoid the complex calculation of the newly captured interface cell, it is modified as follows:

(1) When the vertices of the octahedron reach only the firstorder nearest neighbor liquid cell within time step $\Delta t$, the vertex at this time is used as the growth center of the newly captured interface cell, as shown in Fig. 2.

(2) In a time step $\Delta t$, while the first-order nearest neighbor liquid cell and the second-order neighbor liquid cell are touched at the same time, the vertex at this time is used as the growth center of the two newly captured interface cells. However, the growth rate is still calculated according to the undercooling of their respective cells.
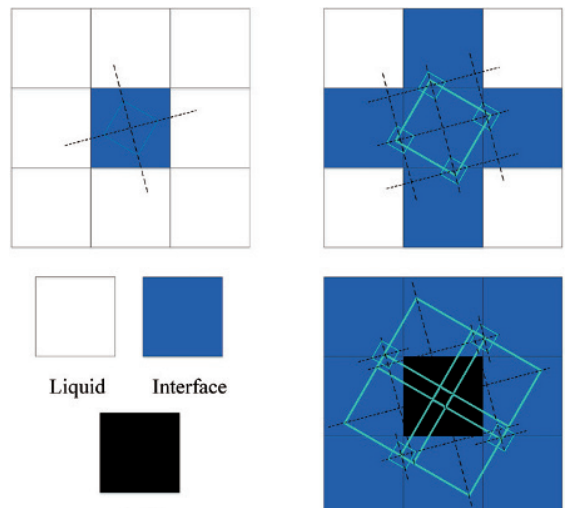

Solid

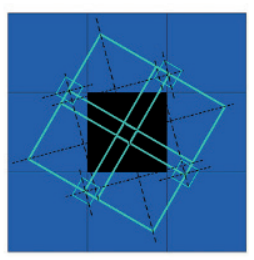

Fig. 2: Schematic diagram of modified decentered square algorithm

\section{Results and discussion}

The material properties of DD406 (Ni-3.92 Cr-9.34Co1.85Mo-8.88W-5.92Al-7.49Ta-2.0RE, mass fraction wt.\%) is calculated by using commercial JMatPro software. The material properties and model parameters used in the simulations are given in Table 1.

\subsection{Dendritic morphology and dendritic spacing}

To verify the correctness of the simulation, the dendrite 
Table 1: Material properties of DD406 superalloy and model parameters used in simulations

\begin{tabular}{lc}
\multicolumn{1}{c}{ Parameter } & Value \\
\hline Density $\left(\mathrm{kg} \cdot \mathrm{m}^{-3}\right)$ & 7223.48 \\
Specific heat capacity $\left(\mathrm{J} \cdot \mathrm{kg}^{-1} \cdot \mathrm{K}^{-1}\right)$ & 1074.60 \\
Thermal conductivity $\left(\mathrm{W} \cdot \mathrm{m}^{-1} \cdot \mathrm{K}^{-1}\right)$ & 37.43 \\
Pouring temperature $(\mathrm{K})$ & 1650 \\
Latent heat $\left(\mathrm{J} \cdot \mathrm{kg}^{-1}\right)$ & 233371.59 \\
Liquidus temperature $(\mathrm{K})$ & 1640.96 \\
Solidus temperature $(\mathrm{K})$ & 1611.47 \\
Initial solute equivalent concentration $(\mathrm{wt} . \%)$ & 31.74 \\
Equivalent coefficient of solute redistribution & 0.57 \\
Equivalent slope of liquidus & -1.96 \\
Eutectic point equivalent concentration $(\mathrm{wt} . \%)$ & 46.80 \\
Gibbs Thomson Coefficient $(\mathrm{K} \cdot \mathrm{m})$ & $1.0 \times 10^{-7}$ \\
Liquid diffusion coefficient $\left(\mathrm{m}^{2} \cdot \mathrm{s}^{-1}\right)$ & $3.0 \times 10^{-9}$ \\
Solid diffusion coefficient $\left(\mathrm{m}^{2} \cdot \mathrm{s}^{-1}\right)$ & $3.0 \times 10^{-12}$ \\
Stefan-Boltzmann radiation constant & $5.672 \times 10^{-8}$ \\
Emissivity coefficient of alloy & 0.89 \\
Emissivity coefficient of shell mould & 0.75 \\
Emissivity coefficient of mold & 0.83 \\
Initial nucleation base number $\left(\mathrm{m}^{-3}\right)$ & $5 \times 10^{8}$ \\
Undercooling variance in nucleation $(\mathrm{K})$ & 0.1 \\
Undercooling mean in nucleation $(\mathrm{K})$ & 0.5 \\
Cell size $(\mu \mathrm{m})$ & 1 \\
\hline
\end{tabular}

evolution of directional solidification under the boundary conditions of 6 crystal seeds $(n)$ with a temperature gradient $(G)$

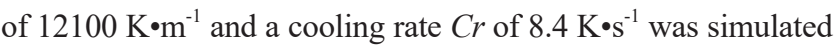
and analyzed, as shown in Fig. 3.

Figure 3 shows that the initial spacing of seed is equivalent to the primary dendritic arm spacing at the initial time. But with the increase of the undercooling, the secondary dendrites are formed on the trunk of dendrite, even the tertiary dendrite arms are also formed on the secondary dendritic arms. The tertiary dendrite arms lead to a decrease in the primary dendritic arm spacing, as shown in Fig. 3 (a) and (b). Besides, the competition

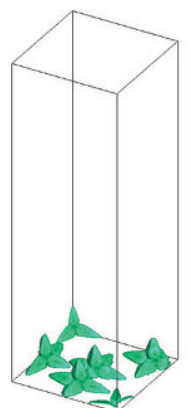

(a) $t=1.0 \mathrm{~s}$

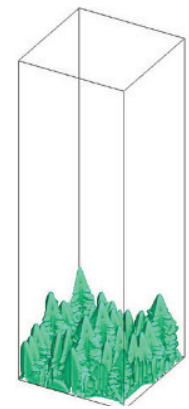

(b) $t=1.5 \mathrm{~s}$

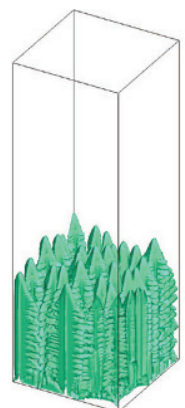

(c) $t=2.0 \mathrm{~s}$ (d) $t=2.5 \mathrm{~s}$

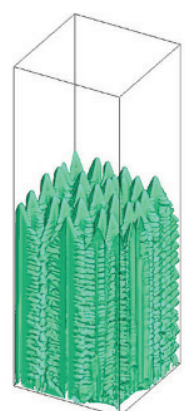

Fig. 3: Dendritic morphology evolution during directional solidification at $n=6, G=12,100 \mathrm{~K} \cdot \mathrm{m}^{-1}$ and $\mathrm{Cr}=8.4 \mathrm{~K} \cdot \mathrm{s}^{-1}$ growth between dendrites can lead to the increase of local primary dendrite spacing, as shown in Fig. 3 (b) and (c). This is caused by the fluctuation of the influencing factors of the solidification process, such as solute composition, temperature, etc. Finally, at $t=2.5 \mathrm{~s}$, the height of the primary dendritic arms is approximately the same. It is considered that the dendritic growth tends to be stable and the size of the primary dendritic spacing of the subsequent dendritic growth is approximately constant. It can be concluded that the growth behavior of dendrites is not only dependent on the parameters of initial solidification boundary conditions, but also related to the results of competitive growth history, which is consistent with the relevant theoretical and experimental conclusions ${ }^{[22]}$.

In order to quantitatively study the dendritic growth, the average spacing of primary dendrites and secondary dendrites were simulated and calculated under the boundary conditions such as initial spacing, temperature gradient and cooling rate. According to the classical primary dendritic arm spacing models of Hunt, KurzFisher and Trivedi ${ }^{[23,24]}$ and the secondary dendritic arm spacing of Feurer-Wunderlin, the dendritic growth is analyzed and compared. The comparison between the simulation results and the theoretical calculation results are shown in Fig. 4 and Fig. 5.

From Fig. 4 and 5, it is known that the simulation results of primary dendritic arm spacing and secondary dendritic arm spacing are consistent with those of classical theory, which show that the proposed and developed model and algorithm are feasible.

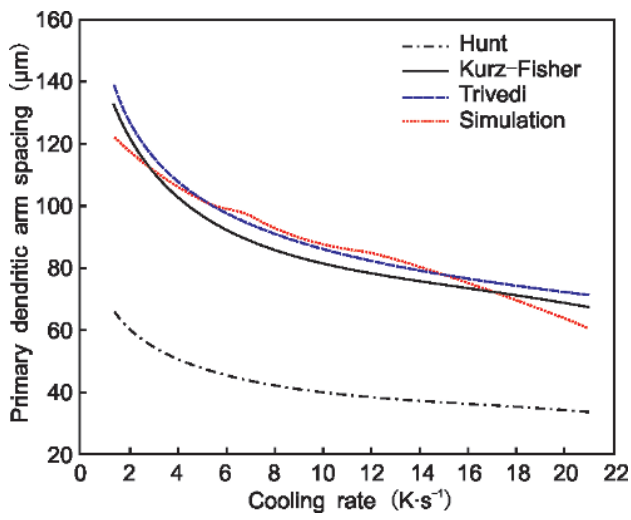

Fig. 4: Comparison of primary dendritic arm spacing with various cooling rate between simulations and theoretical results

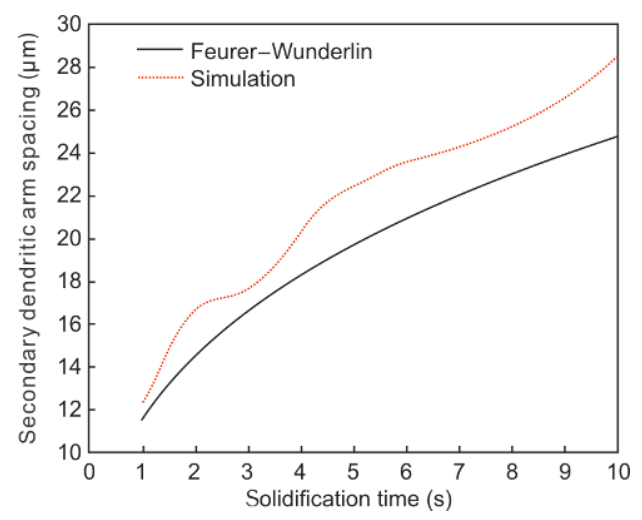

Fig. 4: Comparison of secondary dendritic arm spacing with solidification time between simulations and theoretical results 


\subsection{Evaluation of grain growth simulation}

The same calculation model of dendritic tip growth rate is used in the microscopic dendrite simulation and mesoscopic grain simulation. Based on the same parameters and boundary conditions, the evolution of solidification microstructure between grain and dendrite simulation are compared and analyzed. The mesoscopic grain growth and the microscopic dendrite growth at temperature gradient of $12,100 \mathrm{~K} \cdot \mathrm{m}^{-1}$ and cooling rate of $14 \mathrm{~K} \cdot \mathrm{s}^{-1}$ are shown in Fig. 6 and 7, respectively.

Figure 6 and 7 show that, at the beginning of grain or dendritic growth, the growth rate of grain or dendrites is fast, and it is decreases gradually. This is because the grain or dendrite growth behavior is impeded by the boundary of computation domain. Finally, the two grains or dendrites with orientation a1 and a 2 are obtained. It appears that the mesoscopic grain simulation can be used to obtain results similar to those of microscopic dendrites simulation. But the biggest difference between the two is that the growth time of mesoscopic grain does not match the time of microscopic dendrite growth, that is to say, the growth rate is faster in the mesoscopic grain simulation. The reason is that the mesoscopic grain growth rate is obtained by the KGT model fitting under the supercooling function. But the model is only suitable for describing the stable growth and evolution of the equiaxed crystal of the ideal unrestricted region. Besides, the solute concentration has an important influence on the evolution of solidification microstructure. In the present work, the computation domain is limited. The tip growth rate of each grain is unstable, which leads to the difference of the results. Therefore, in order to accurately simulate the growth behavior of the mesoscopic grain, the growth rate of the dendritic tip of the mesoscopic grain must be calculated more accurately.

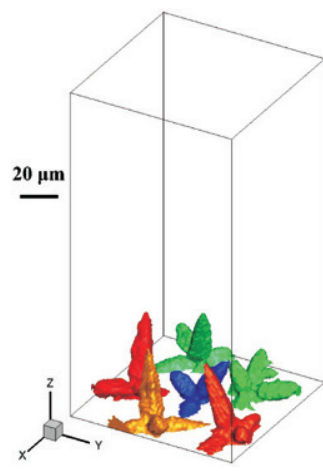

(a) $t=0.1 \mathrm{~s}$

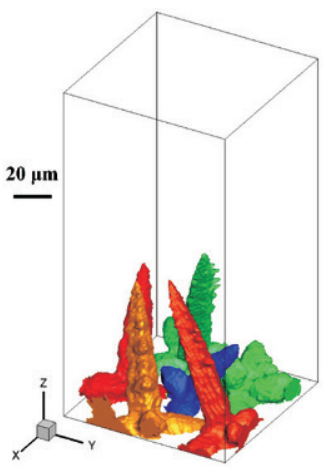

(b) $t=0.2 \mathrm{~s}$

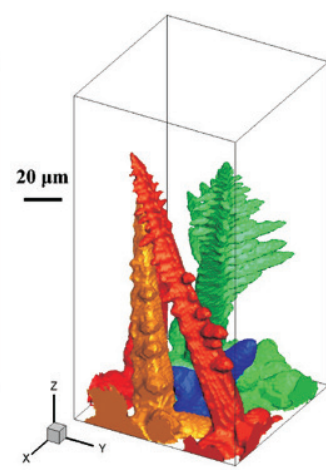

(c) $t=0.3 \mathrm{~s}$

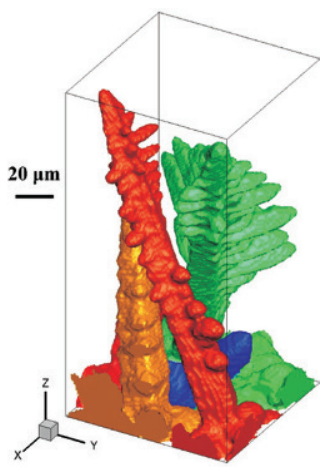

(d) $t=0.4 \mathrm{~s}$

Fig. 6: Microscopic dendritic growth during directional solidification at $n=6, G=12,100 \mathrm{~K}^{\prime} \mathrm{m}^{-1}$ and $\mathrm{Cr}=14 \mathrm{~K} \cdot \mathrm{s}^{-1}$

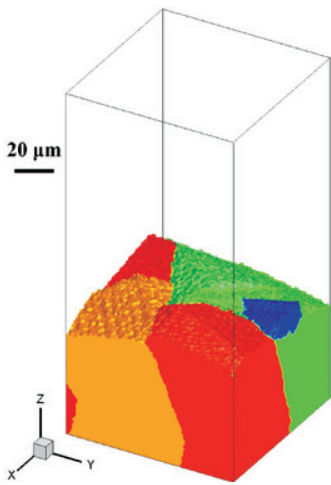

(a) $t=0.01 \mathrm{~s}$

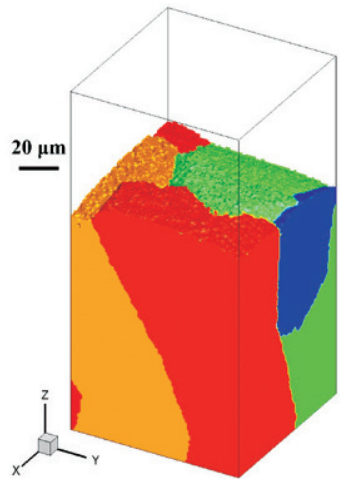

(b) $t=0.03 \mathrm{~s}$

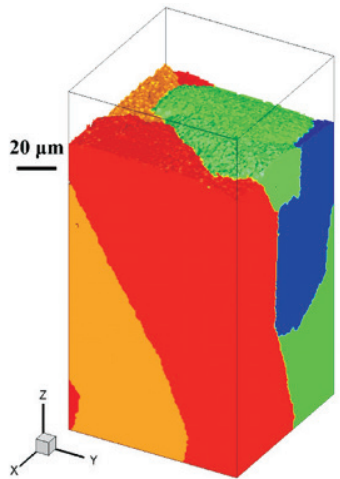

(c) $t=0.05 \mathrm{~s}$

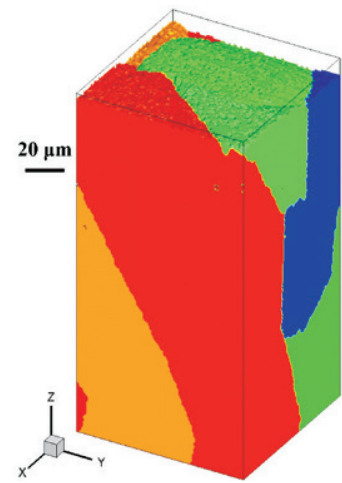

(d) $t=0.07 \mathrm{~s}$

Fig. 7: Mesoscopic grain growth during directional solidification at $n=6, G=12100 \mathrm{~K} \cdot \mathrm{m}^{-1}$ and $\mathrm{Cr}=14 \mathrm{~K} \cdot \mathrm{s}^{-1}$

\subsection{Simulation of directional solidification process of single crystal superalloy}

In the directional solidification process of superalloy, the drawing speed is the key process parameter. The directional solidification process of single crystal superalloy under different drawing speeds of 6,9 and $12 \mathrm{~mm} \cdot \mathrm{min}^{-1}$ are simulated by the mesoscopic grain simulation method. In order to accurately simulate the mesoscopic grain growth, the mesoscopic grain growth rate is obtained indirectly by the fitting function of the average growth rate in the microscopic dendritic growth simulation.
The distribution of the temperature field, the solid-liquid interface and the mesoscopic grain structure during the directional solidification are shown in Fig. 8.

Figure 8 shows that the temperature gradient of the spiral selector is approximately along the $Z$-axis direction at the initial time, when the solid-liquid interface enters the spiral selector, and its temperature gradient is similar to the helix centerline of the spiral selector. But at time $t=590 \mathrm{~s}$, it can be seen that the solid-liquid interface is no longer along the helix centerline of the spiral selector, but forms the mushy zone near the 


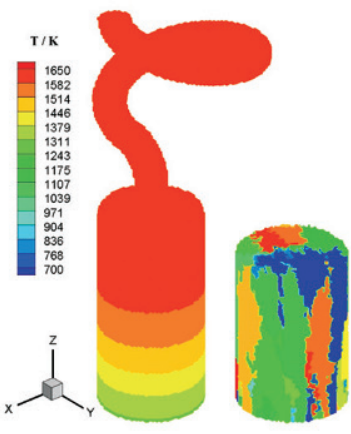

(a) $t=250 \mathrm{~s}$

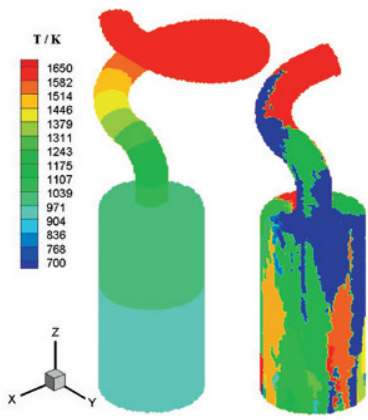

(c) $t=510 \mathrm{~s}$
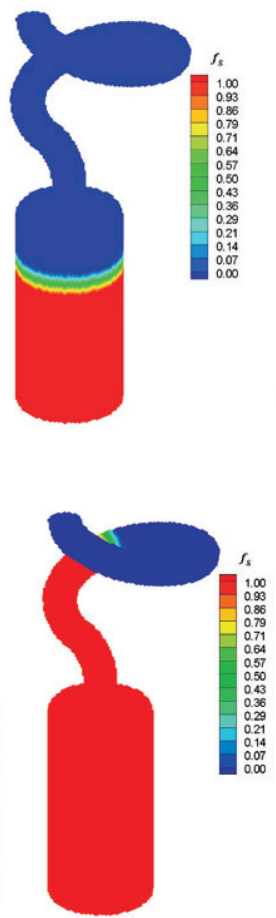
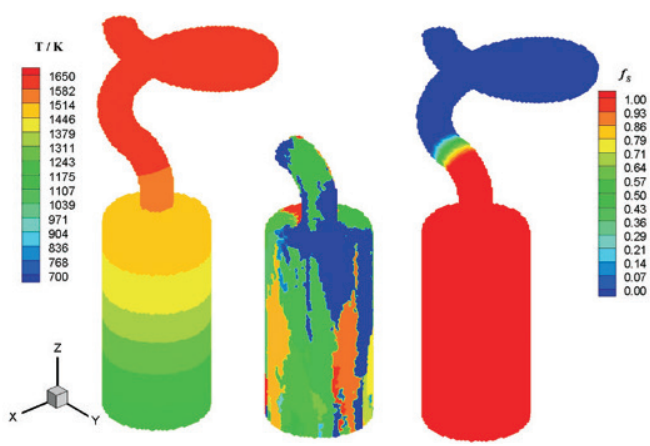

(b) $t=430 \mathrm{~s}$

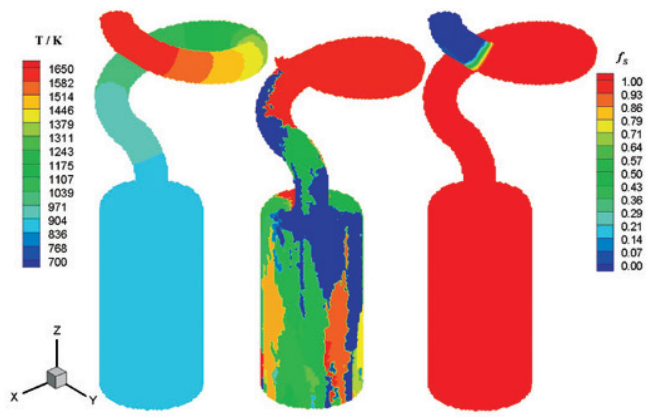

(d) $t=590 \mathrm{~s}$

Fig.8: Evolution of temperature field, solid-liquid interface and mesoscopic grain structure during directional solidification at $6 \mathrm{~mm} \cdot \mathrm{min}^{-1}$

central wall, which may affect the changes in the solidification microstructure. Finally, due to the efficient selection of the spiral selector, only one grain in the solidification microstructure grows along the spiral selector and forms a single crystal.

In order to further quantitatively characterize the growth and the selection efficiency of the directional solidification microstructure

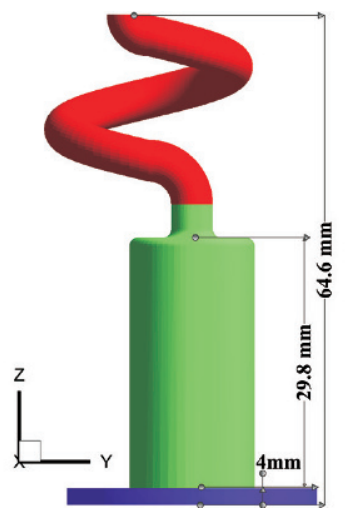

(a) Geometry model

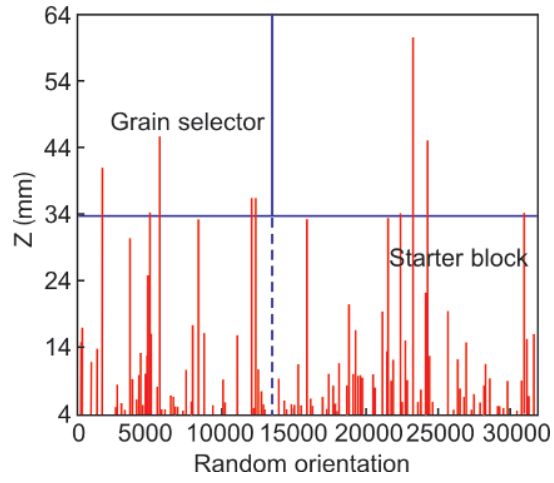

(d) $9 \mathrm{~mm} \cdot \mathrm{min}^{-1}$ along the $Z$-axis at different drawing speeds, the grains number along the $Z$-axis direction is analyzed. Because of the randomness of nucleation, the grain orientation is characterized by a random integer. The maximum integer is taken as the maximum grain number. The growth distance along the $Z$ axis of different grains at different drawing speeds is shown in Fig. 9.

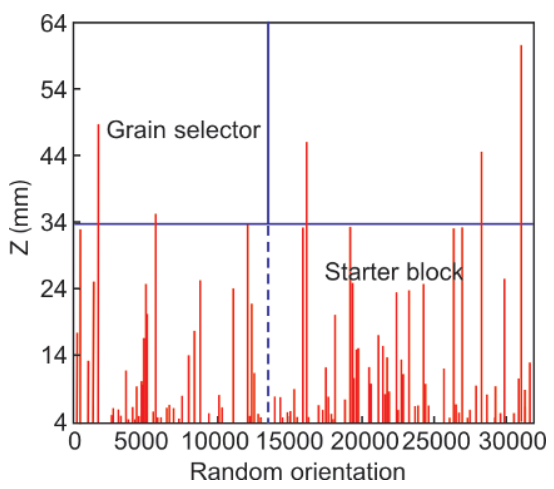

(b) $6 \mathrm{~mm} \cdot \mathrm{min}^{-1}$

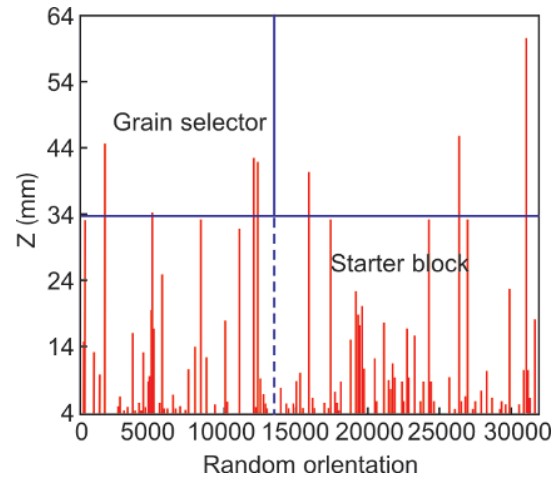

(d) $12 \mathrm{~mm} \cdot \mathrm{min}^{-1}$

Fig. 9: Growth distance along $Z$ axis of different grains at different drawing speeds 
From Fig. 9, most of the grains can be eliminated in the starter block. At drawing speeds of 6,9 , and $12 \mathrm{~mm} \bullet \mathrm{min}^{-1}$, there are 5 , 8 and 7 grains with different crystallographic orientations that can be selected out respectively. That is to say, reducing the drawing speed can cut down the number of grains selected from the starter block, thus improving the crystal selection efficiency.

\section{Conclusions}

In order to quickly and accurately predict the evolution of solidification microstructure, a three-dimensional cellular automaton model coupled dendritic scale and grain scale is developed, and a macro-mesoscopic/microscopic coupling solution algorithm is proposed. The nickel-based single crystal superalloy DD406 was researched and the conclusions can be drawn as follows:

(1) The results of the dendritic scale simulation are consistent with the theoretical and experimental results given in previous studies. It can be considered that the developed threedimensional cellular automaton model is feasible.

(2) Even though the growth time of mesoscopic grain does not match the time of microscopic dendrite growth, similar results can be obtained from the two kinds of simulations.

(3) The parameters of drawing speed and geometry size of starter block and grain selector all can affect the solidification microstructure. Under the same nucleation and random orientation, the final growth distance along the $Z$ axis of different grains at different drawing speeds is similar.

\section{References}

[1] Li Y, Liu L, Huang T, et al. Simulation of stray grain formation in Ni-base single crystal turbine blades fabricated by HRS and LMC techniques. China Foundry, 2017, 14(2): 75-79.

[2] Meng X, Li J, Zhu S, et al. Method of stray grain inhibition in the platforms with different dimensions during directional solidification of a ni-base superalloy. Metallurgical and Materials Transactions A: Physical Metallurgy and Materials Science, 2014, 45(3): 1230-1237.

[3] Cao L, Liao D, Lu Y, et al. Heat transfer model of directional solidification by Imc process for superalloy casting based on finite element method. Metallurgical and Materials Transactions A: Physical Metallurgy and Materials Science, 2016, 47(9): 4640-4647.

[4] Zhang H, Xu Q. Multi-scale simulation of directional dendrites growth in superalloys. Journal of Materials Processing Technology, 2016, 238: 132-141.

[5] Szeliga D, Kubiak K, Motyka M, et al. Directional solidification of Ni-based superalloy castings: Thermal analysis. Vacuum, 2016, 131: 327-342.

[6] Zhi X, Liu J, Xing J, et al. Effect of cerium modification on microstructure and properties of hypereutectic high chromium cast iron. Materials Science and Engineering: A, 2014, 603: 98-103.
[7] Stefanescu D M. Science and Engineering of Casting Solidification. Switzerland: Springer, 2015.

[8] Gandin C, Desbiolles J, Rappaz M, et al. A three-dimensional cellular automaton-finite element model for the prediction of solidification grain structures. Metallurgical and Materials Transactions A, 1999, 30(12): 3153-3165.

[9] Gandin C, Rappaz M. A 3D Cellular Automaton algorithm for the prediction of dendritic grain growth. Acta Materialia, 1997, 45(5): 2187-2195.

[10] Carozzani T, Digonnet H, Bellet M, et al. 3D CAFE simulation of a macrosegregation benchmark experiment. IOP Conference Series: Materials Science and Engineering, 2012, 33(1): 12087-12096.

[11] Guillemot G, Gandin C, Combeau H, et al. A new cellular automaton-finite element coupling scheme for alloy solidification. Modelling and Simulation in Materials Science and Engineering, 2004, 12(3): 545-556.

[12] Carter P, Cox D C, Gandin C A, et al. Process modelling of grain selection during the solidification of single crystal superalloy castings. Materials Science and Engineering A, 2000, 280(2): 233-246.

[13] Meng X, Lu Q, Li J, et al. Modes of grain selection in spiral selector during directional solidification of nickel-base superalloys. Journal of Materials Science \& Technology, 2012, 28(3): 214-220.

[14] Xu Q, Zhang H, Qi X, et al. Multiscale Modeling and Simulation of Directional Solidification Process of Turbine Blade Casting with MCA Method. Metallurgical and Materials Transactions B, 2014, 45(2): 555-561.

[15] Nastac L. Numerical modeling of solidification morphologies and segregation patterns in cast dendritic alloys. Acta Materialia, 1999, 47(17): 4253-4262.

[16] Zhu M, Stefanescu D. Virtual front tracking model for the quantitative modeling of dendritic growth in solidification of alloys. Acta Materialia, 2007, 55(5): 1741-1755.

[17] Pan S, Zhu M. A three-dimensional sharp interface model for the quantitative simulation of solutal dendritic growth. Acta Materialia, 2010, 58(1): 340-352.

[18] Zhang $\mathrm{X}$, Zhao J, Jiang $\mathrm{H}$, et al. A three-dimensional cellular automaton model for dendritic growth in multi-component alloys. Acta Materialia, 2012, 60(5): 2249-2257.

[19] Yuan L, Lee P D. A new mechanism for freckle initiation based on microstructural level simulation. Acta Materialia, 2012, 60(12): 4917-4926.

[20] Rappaz M, Gandin C A. Probabilistic modelling of microstructure formation in solidification processes. Acta Metallurgica et Materialia, 1993, 41(2): 345-360.

[21] Nastac L, Stefanescu D M. An analytical model for solute redistribution during solidification of planar, columnar, or equiaxed morphology. Metallurgical Transactions A, 1993, 24(9): 2107-2118.

[22] Wang W, Lee P D, Mclean M. A model of solidification microstructures in nickel-based superalloys: predicting primary dendrite spacing selection. Acta Materialia, 2003, 51(10): 2971-2987.

[23] Kurz W, Fisher D J. Dendrite growth at the limit of stability: tip radius and spacing. Acta Metallurgica, 1981, 29(1): 11-20.

[24] Trivedi R. Interdendritic Spacing: Part II. A Comparison of Theory and Experiment. Metallurgical Transactions A, 1984, 15(6): 977-982. 